\title{
A new determination of the charm mass from the non-analytic reconstruction of the heavy quark correlator
}

\author{
David Greynat ${ }^{* \dagger}$ \\ Departamento de Física Teórica, Facultad de Ciencias \\ Universidad de Zaragoza \\ 50009 Zaragoza, Spain \\ E-mail: david.greynat@gmail.com
}

Pere Masjuan

Institut für Kernphysik,

Johannes Gutenberg-Universität

J.J. Becher-Weg 45

D-55099 Mainz, Germany

E-mail: mas juan@kph.uni-mainz.de

\begin{abstract}
Using the new non-analytic reconstruction method obtained from Mellin-Barnes properties, one can extract the value $m_{c}(\overline{\mathrm{MS}})=1.12 \pm 0.08 \mathrm{GeV}$ from experimental data of the radiationcorrected measured hadronic cross section to the calculated lowest-order cross section for muon pair production in the heavy-quark approximation.
\end{abstract}

Xth Quark Confinement and the Hadron Spectrum,

October 8-12, 2012

TUM Campus Garching, Munich, Germany

*Speaker.

$\dagger$ This work has been supported by the Spanish DGIID-DGA grant 2009-E24/2, the Spanish MICINN grants FPA2009-09638 and CPAN-CSD2007-00042 and by the Deutsche Forschungsgemeinschaft DFG through the Collaborative Research Center "The Low-Energy Frontier of the Standard Model" (SFB 1044). 


\section{Introduction}

An accurate determination of the charm mass plays an important role on the precise physical evaluation of several observables, from $\mathrm{K}$ and $\mathrm{B}$ decays to CKM matrix elements and in lattice QCD. One of the usual techniques to extract the charm mass is to use the sum rules approach based on the relation between the moments of the production rate $R$ and the inverse power of the square mass of the $c$ quark, and the Padé method (see [1,2]). This approach should confront the fact that one have to employ the moments of the integral of $\mathrm{R}$ over the whole energy range, which are global properties, even though they are only known up to a certain scale $\Lambda$ (since we only know experimentally $R$ in a finite window). We propose to wield the local properties of $R$ through a new "non-analytic reconstruction" method [3,4]. As we will show, this approach allows us to obtain local properties of the heavy quark correlators at each points of the spectrum with a systematic error and then to find a value of the charm mass directly on a $\chi^{2}$ regression on the experimental points.

\section{Details of the method}

\subsection{Non-analytic reconstruction}

Let us consider the vector polarization function

$$
\left(q_{\mu} q_{v}-q^{2} g_{\mu v}\right) \Pi\left(q^{2}\right)=i \int \mathrm{d}^{4} x \mathrm{e}^{i q x}\left\langle 0\left|\mathrm{~T} j_{\mu}(x) j^{\mu}(0)\right| 0\right\rangle,
$$

with the current $j_{\mu}(x)=\bar{\psi}(x) \gamma_{\mu} \psi(x)$, which has a cut in the complex plane starting at $q^{2}=4 m^{2}$, where $m$ is the (pole) mass of the heavy quark considered. In QCD perturbation theory, it can be expanded as

$$
\Pi\left(q^{2}\right)=\Pi(0)+\Pi^{(0)}\left(q^{2}\right)+\left(\frac{\alpha_{s}}{\pi}\right) \Pi^{(1)}\left(q^{2}\right)+\left(\frac{\alpha_{s}}{\pi}\right)^{2} \Pi^{(2)}\left(q^{2}\right)+\left(\frac{\alpha_{s}}{\pi}\right)^{3} \Pi^{(3)}\left(q^{2}\right)+\mathscr{O}\left(\alpha_{s}^{4}\right),
$$

where only $\Pi^{(0)}$ and $\Pi^{(1)}$ are know analytically, (for $z=q^{2} / 4 m^{2}$ )

$$
\Pi^{(0)}(z)=\frac{3}{16 \pi^{2}}\left[\frac{20}{9}+\frac{4}{3 z}-\frac{4(1-z)(1+2 z)}{3 z} G(z)\right]
$$

and

$$
\begin{aligned}
\Pi^{(1)}(z)=\frac{3}{16 \pi^{2}}\left[\frac{5}{6}+\frac{13}{6 z}-\frac{(1-z)(3+2 z)}{z} G(z)+\right. & \frac{(1-z)(1-16 z)}{6 z} G^{2}(z) \\
& \left.-\frac{(1+2 z)}{6 z}\left(1+2 z(1-z) \frac{d}{d z}\right) \frac{I(z)}{z}\right],
\end{aligned}
$$

in which we used the auxiliary functions,

$$
\begin{aligned}
G(z)= & \frac{2 u \log u}{u^{2}-1} \\
I(z)=6 & {\left[\zeta_{3}+4 \operatorname{Li}_{3}(-u)+2 \operatorname{Li}_{3}(u)\right] } \\
& -8\left[2 \operatorname{Li}_{2}(-u)+\operatorname{Li}_{2}(u)\right] \ln u-2[2 \ln (1+u)+\ln (1-u)] \ln ^{2} u,
\end{aligned}
$$


and

$$
u=\frac{\sqrt{1-1 / z}-1}{\sqrt{1-1 / z}+1}
$$

As it has been shown [3, 4] even if the functions $\Pi^{(2)}$ and $\Pi^{(3)}$ are unknown analytically, one can reconstruct them from their expansions around $q^{2} \rightarrow 0$ (Taylor expansion), $q^{2} \rightarrow 4 m^{2}$ (threshold expansion) and $q^{2} \rightarrow \infty$ (OPE), as

$$
\Pi^{(k)}(z)=\sum_{n=0}^{N_{k}^{*}} \Omega^{(k)}(n) \omega^{n}+\sum_{p, \ell}(-)^{\ell}\left[\alpha_{p, \ell}^{(k)} \operatorname{Li}^{(\ell)}(p, \omega)-\beta_{p, \ell}^{(k)} \operatorname{Li}^{(\ell)}(p,-\omega)\right]+\mathscr{E}^{(k)}\left(N_{k}^{*}, \omega\right) .
$$

Let emphasize a little this expression. First one defines the so-called conformal change of variable

$$
z=\frac{4 \omega}{(1+\omega)^{2}} \quad, \quad \omega=\frac{1-\sqrt{1-z}}{1+\sqrt{1-z}} .
$$

This change of variables maps the cut $z$ plane into a unit disc in the $\omega$ plane, as we can see on Figure 2.1. The physical cut $z \in[1, \infty[$ is transformed into the circle $|\omega|=1$. The points $z=0$ into $\omega=0, z=1$ into $\omega=1$, the limit $z \rightarrow+\infty \pm i \varepsilon$ into $\omega \rightarrow-1 \pm i \varepsilon$, and $z \rightarrow-\infty$ into $\omega \rightarrow-1$.
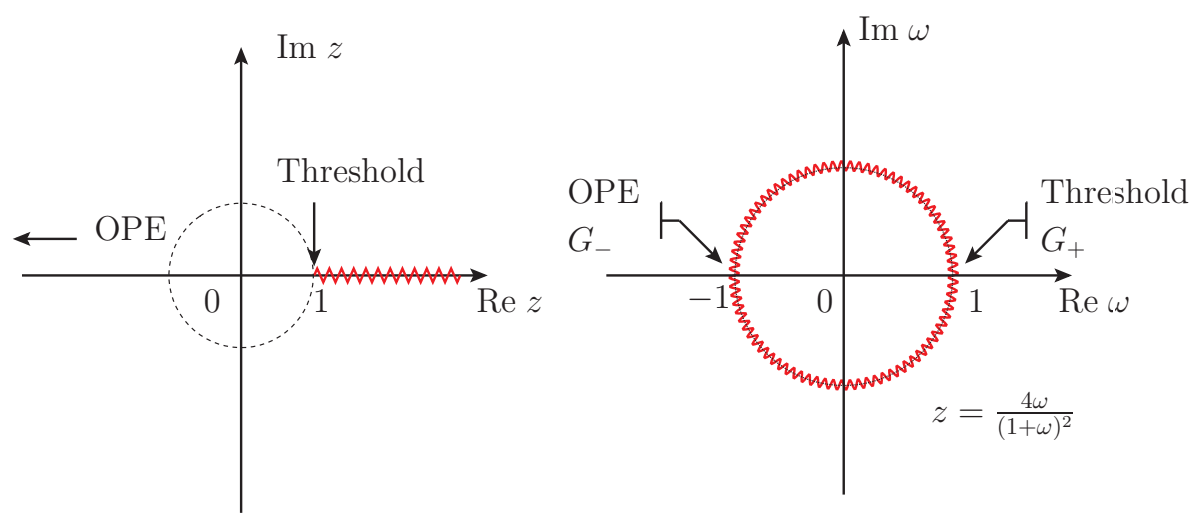

Figure 1: Conformal mapping between $z$ and $\omega$.

For both functions $\Pi^{(2)}$ and $\Pi^{(3)}$, Feynman diagrams calculations at $q^{2} \rightarrow 0$ give the expansions up to an order $N_{k}^{*}$ (for $k=2,3$ )

$$
\Pi^{(k)}(z) \underset{q^{2} \rightarrow 0}{=} \sum_{n=0}^{N_{k}^{*}} C^{(k)}(n) z^{n}+\mathscr{O}\left(z^{N_{k}^{*}+1}\right) \underset{\omega \rightarrow 0}{=} \sum_{n=0}^{N_{k}^{*}} \Omega^{(k)}(n) \omega^{n}+\mathscr{O}\left(\omega^{N_{k}^{*}+1}\right),
$$

where the relation between the two coefficients $C^{(k)}$ and $\Omega^{(k)}(n)$ is

$$
\begin{aligned}
& \Omega^{(k)}(n)=(-1)^{n} \sum_{p=1}^{n} \frac{(-1)^{p} 4^{p} \Gamma(n+p)}{\Gamma(2 p) \Gamma(n+1-p)} C^{(k)}(p), \\
& C^{(k)}(n)=2^{1-2 n} \Gamma(2 n) \sum_{p=1}^{n} \frac{p}{\Gamma(1+n-p) \Gamma(1+n+p)} \Omega^{(k)}(p) .
\end{aligned}
$$


The main part of the approximation in (2.8) lies on the combination of the polylogarithms functions,

$$
\operatorname{Li}^{(\ell)}(s, \omega)=\frac{\mathrm{d}^{\ell}}{\mathrm{d} s^{\ell}}\left[\frac{\omega}{\Gamma(s)} \int_{0}^{1} \frac{\mathrm{d} t}{1-\omega t} \log ^{s-1}\left(\frac{1}{t}\right)\right] \underset{|\omega|<1}{=}(-1)^{\ell} \sum_{n=1}^{\infty} \frac{\log ^{\ell} n}{n^{s}} \omega^{n}
$$

and the analytic evaluation of the coefficients $\alpha_{p, \ell}^{(k)}$ and $\beta_{p, \ell}^{(k)}$. In order to reconstruct $\Pi^{(2)}$ and $\Pi^{(3)}$, we collect here their corresponding coefficients (see $[3,4]$ for more details)

$$
\begin{aligned}
& \left\{\begin{array}{l}
\alpha_{0,0}^{(2)}=3.44514 \\
\alpha_{1,0}^{(2)}=-0.492936 \\
\alpha_{1,1}^{(2)}=2.25 \\
\alpha_{2,0}^{(2)}=3.05433
\end{array}, \quad\left\{\begin{array}{l}
\beta_{1,0}^{(2)}=0.33723 \\
\beta_{1,1}^{(2)}=0.211083 \\
\beta_{3,0}^{(2)}=0.183422 \\
\beta_{3,1}^{(2)}=-0.620598
\end{array},\right.\right. \\
& \left\{\begin{array}{l}
\alpha_{-1,0}^{(3)}=10.5456 \\
\alpha_{0,1}^{(3)}=31.0063 \\
\alpha_{0,0}^{(3)}=-11.0769 \\
\alpha_{1,0}^{(3)}=36.3318 \\
\alpha_{1,1}^{(3)}=37.1514 \\
\alpha_{1,2}^{(3)}=10.125
\end{array}, \quad\left\{\begin{array}{l}
\beta_{1,0}^{(3)}=-0.181866 \\
\beta_{1,1}^{(3)}=0.211083 \\
\beta_{1,2}^{(3)}=-0.879515 \\
\beta_{3,0}^{(3)}=-10.4385 \\
\beta_{3,2}^{(3)}=3.82702
\end{array},\left\{\begin{array}{l}
\beta_{5,0}^{(3)}=-70.9277 \\
\beta_{5,1}^{(3)}=56.3093 \\
\beta_{5,2}^{(3)}=20.9951 \\
\beta_{5,3}^{(3)}=-7.55063
\end{array} .\right.\right.\right.
\end{aligned}
$$

At least, one gives the error functions $\mathscr{E}^{(k)}$,

$$
\begin{aligned}
& \mathscr{E}^{(2)}\left(N_{2}^{*}, \omega\right)=\left[\begin{array}{c}
+1 \\
0
\end{array}\right] \sum_{n=N_{2}^{*}+1}^{\infty} \frac{\log ^{1.5} n}{n^{3}} \omega^{n} \\
& \mathscr{E}^{(3)}\left(N_{3}^{*}, \omega\right)=\left[\begin{array}{c}
+15 \\
-15
\end{array}\right] \sum_{n=N_{3}^{*}+1}^{\infty} \frac{\log ^{3} n}{n^{2}} \omega^{n},
\end{aligned}
$$

which encode the systematic error from the reconstructions.

\subsection{Experimental data}

There exists several experimental results for the $e^{+} e^{-}$in hadrons that one can use for the fitting of the $c$ quark mass. Each of the experiments give the ratio $R(s)$ of the radiation-corrected measured hadronic cross section to the calculated lowest-order cross section for muon pair production,

$$
R(s)=\frac{\sigma_{0}\left(e^{+} e^{-} \longrightarrow \text { hadrons }\right)}{\sigma_{0}\left(e^{+} e^{-} \longrightarrow \mu^{+} \mu^{-}\right)}=\frac{\sigma_{0}\left(e^{+} e^{-} \longrightarrow \text { hadrons }\right)}{4 \pi \alpha^{2} / 3 s},
$$

that has the experimental values shown in Fig. 2 .

This Fig 2 shows that the complete spectrum is sensitive to resonances, as expected. It is obvious that a perturbative approach cannot take into account the resonances description, then one 


\begin{tabular}{|c|c|}
\hline \hline Experiment & Reference \\
\hline MARK I & {$[5]$} \\
PLUTO & {$[6]$} \\
CrystalBall (Run 1) & {$[7]$} \\
CrystalBall (Run 2) & {$[7]$} \\
MD1 & {$[8]$} \\
CLEO & {$[9]$} \\
CLEO & {$[10,11]$} \\
BES & {$[12]$} \\
BES & {$[13]$} \\
CLEO & {$[14]$} \\
CLEO & {$[15]$} \\
\hline \hline
\end{tabular}

Table 1: All different experimental sets considered for the fits.

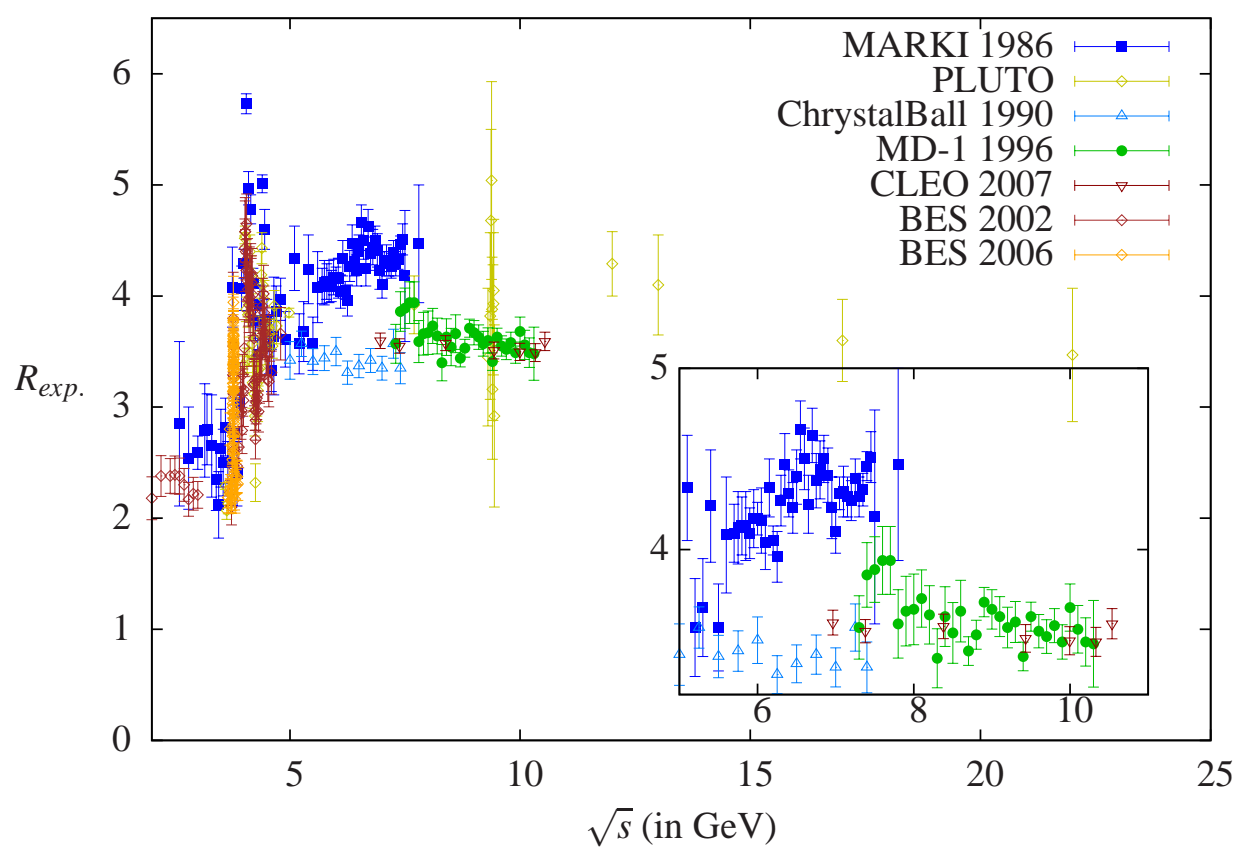

Figure 2: Collection of the different experimental sets for the V-V spectrum.

has to make an arbitrary choice on where we assume that the continuum limit is reached or in other words, where the perturbative description is pertinent. Let's choose the value of $5 \mathrm{GeV}$. Of course the influence of the arbitrariness has to be discussed and taken account in the evaluation of the error but it is something depending on the perturbative and heavy-quark limit more than the reconstruction itself.

The idea now is to perform a fit among all this data points to extract the perturbative mass $m_{c}$ of the $c$-quark. 


\subsection{Fitting approach}

The first step in the fitting procedure is to choose the following expression for the running $\alpha_{s}(s)$,

$$
\begin{aligned}
\alpha_{s}(s)=\frac{4 \pi}{\beta_{0} \ln \left(s / \Lambda^{2}\right)}\left[1-\frac{2 \beta_{1}}{\beta_{0}^{2}}\right. & \frac{\ln \left[\ln \left(s / \Lambda^{2}\right)\right]}{\ln \left(s / \Lambda^{2}\right)} \\
& \left.+\frac{4 \beta_{1}^{2}}{\beta_{0}^{4} \ln ^{2}\left(s / \Lambda^{2}\right)}\left(\left(\ln \left[\ln \left(s / \Lambda^{2}\right)\right]-\frac{1}{2}\right)^{2}+\frac{\beta_{2} \beta_{0}}{8 \beta_{1}^{2}}-\frac{5}{4}\right)\right],
\end{aligned}
$$

where $\Lambda$ is the energy scale and the $\beta$-function has coefficients

$$
\beta_{0}=11-\frac{2 n_{f}}{3}, \quad \beta_{1}=51-\frac{19 n_{f}}{3}, \quad \beta_{2}=2857-\frac{5033 n_{f}}{9}+\frac{325 n_{f}^{2}}{27},
$$

and $n_{f}$ is the number of quarks with mass smaller than $\sqrt{s} / 2$.

The theoretical expression (2.18) is related to $\Pi\left(q^{2}\right)(2.2)$, up to $\alpha_{s}^{3}$,

$$
\begin{aligned}
R_{\text {th. }}(s)= & {\left[\left(\frac{2}{3}\right)^{2}+\left(\frac{1}{3}\right)^{2}+\left(\frac{1}{3}\right)^{2}\right] N_{c}\left[1+\frac{\alpha_{s}(s)}{\pi}+1.525\left(\frac{\alpha_{s}(s)}{\pi}\right)^{2}-11.686\left(\frac{\alpha_{s}(s)}{\pi}\right)^{3}\right] } \\
& +12 \pi\left(\frac{2}{3}\right)^{2} \operatorname{Im}\left[\Pi^{(0)}+\frac{4}{3} \frac{\alpha_{s}(s)}{\pi} \Pi^{(1)}+\left(\frac{\alpha_{s}(s)}{\pi}\right)^{2} \Pi^{(2)}+C_{3}\left(\frac{\alpha_{s}(s)}{\pi}\right)^{3} \Pi^{(3)}\right]
\end{aligned}
$$

where all $\Pi^{(k)}$ functions have the argument $z=\frac{s}{4 m_{c}^{2}}$, and $N_{c}$ is the number of colors.

The goal of the analysis is to extract $m_{c}$ from the comparison between the value of $R_{\text {exp. }}$ and $R_{\text {th. }}$. The usual method used is to built the moments associated to $\mathrm{R}$ from 0 to $\Lambda^{2}$ and identifying the coefficients of the Taylor expansion that are proportional up to a factor to $m_{c}^{-2}$. Instead of this approach, we propose to perform the analysis directly on the function itself, because thanks to the reconstruction method formula (2.8), its expression is available and its systematic error too (2.16).

For this we will use a $\chi^{2}$-method with the assumption

$$
\chi^{2}\left(m_{c}\right) \doteq \sum_{j=1}^{N}\left(\frac{R_{\text {exp. }}\left(s_{j}\right)-R_{\text {th. }}\left(s_{j}\right)}{\sigma_{\text {exp. }}\left(s_{j}\right)}\right)^{2}+\left(\frac{R_{\text {exp. }}\left(s_{j}\right)-R_{\text {th. }}\left(s_{j}\right)}{\sigma_{\text {th. }}\left(s_{j}\right)}\right)^{2},
$$

where the $s_{j}$ are the experimental energy points, the $\sigma_{\text {exp. }}$ is the experimental error and the theoretical error $\sigma_{\text {th. }}$ due the approximation of the reconstruction is given by

$$
\begin{aligned}
\sigma_{\text {th. }}^{2}(s)=\frac{256 \pi^{2}}{9}\left|\operatorname{Im}\left[\left(\frac{\alpha_{s}(s)}{\pi}\right)^{2} \mathscr{E}^{(2)}\left(N_{2}^{*}, \omega\right)\right]\right|^{2} \\
+\frac{256 \pi^{2}}{9} C_{3}^{2}\left|\operatorname{Im}\left[\left(\frac{\alpha_{s}(s)}{\pi}\right)^{3} \mathscr{E}^{(3)}\left(N_{3}^{*}, \omega\right)\right]\right|^{2},
\end{aligned}
$$

with $\omega=\frac{1-\sqrt{1-\frac{s}{4 m_{c}^{2}}}}{1+\sqrt{1-\frac{s}{4 m_{c}^{2}}}}$ 


\section{Results}

\subsection{Numerical results at order $\alpha_{s}^{2}$}

At $\alpha_{s}^{2}$ order, one obtains after a regression procedure with a $\chi_{\min }^{2} /$ d.o.f. $=1.03$,

$$
m_{c}(\text { pole })=1.85 \pm 0.08 \mathrm{GeV},
$$

that is translated into the $\overline{\mathrm{MS}}$ mass as [16]

$$
m_{c}(\overline{\mathrm{MS}})=1.12 \pm 0.08 \mathrm{GeV} .
$$

Assuming now that the mass $m_{c}$ obeys to a Gaussian density of probability, one can easily reconstruct points by points the error generated on $R_{\text {th. }}$ by this hypothesis, taking into account that the relation between $m_{c}$ and $R_{\mathrm{th}}$ is highly non linear and non trivial for expressing the error. We choose then to use a Monte-Carlo approach to obtaining the mean value of $R_{\text {th. }}$ and its error as shown in Fig 3.

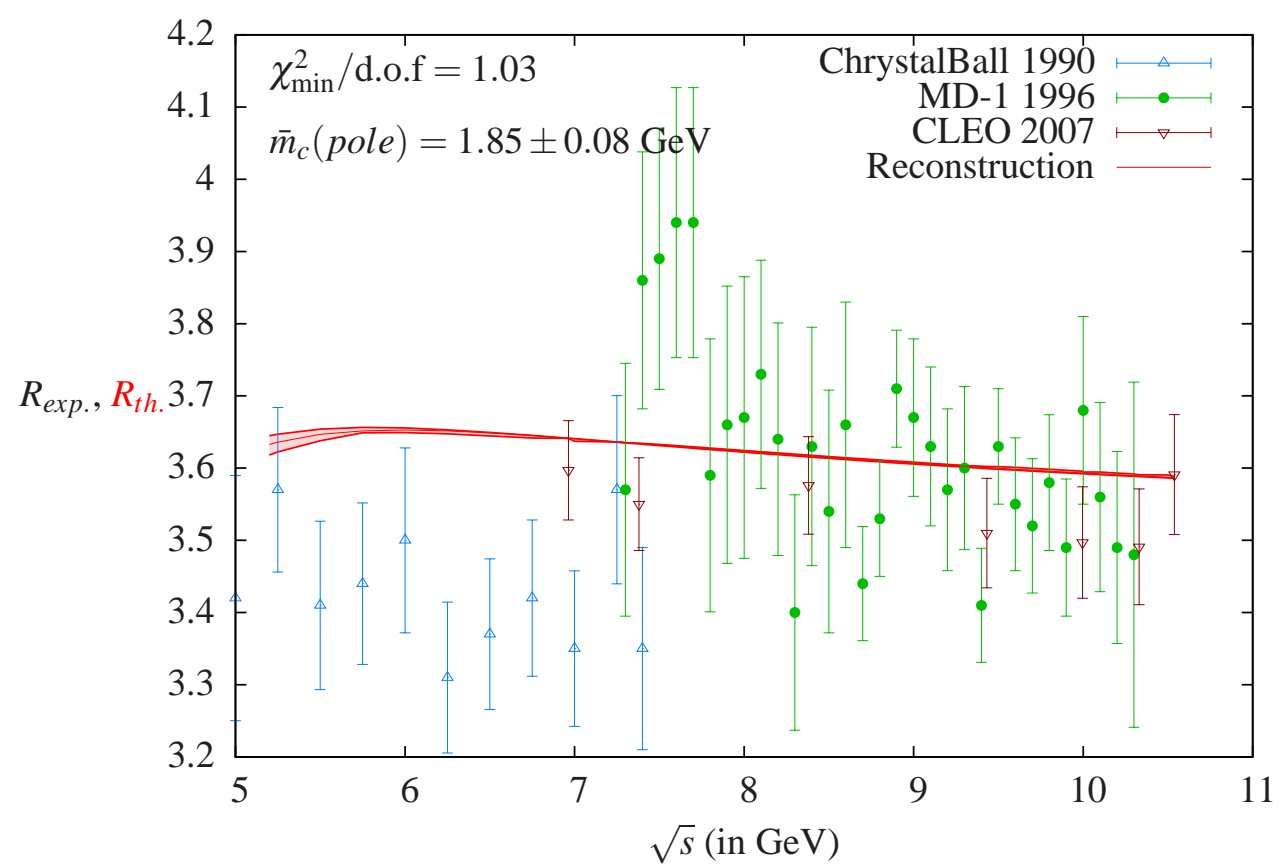

Figure 3: The reconstructed radiation-corrected measured hadronic cross section to the calculated lowestorder cross section for muon pair production.

\section{Conclusions}

We show that it is possible to extract the charm mass value after a $\chi^{2}$ regression to the experimental data of the radiation-corrected measured hadronic cross section to the calculated lowestorder cross section for muon pair production using the non-analytic reconstruction of the heavyquark correlators. We present here a preliminary result up to $\alpha_{s}^{2}$. The next step would include the order $\alpha_{2}^{3}$ and a complete analysis of all different systematic contributions [17]. 


\section{References}

[1] B. Dehnadi, A. H. Hoang, V. Mateu and S. M. Zebarjad, "Charm Mass Determination from QCD Charmonium Sum Rules at Order $\alpha_{s}^{3 ",}$ arXiv:1102.2264 [hep-ph].

[2] P. Masjuan and S. Peris, "Pade Theory applied to the vacuum polarization of a heavy quark," Phys. Lett. B 686 (2010) 307.

[3] D. Greynat and S. Peris, "Resummation of Threshold, Low- and High-Energy Expansions for Heavy-Quark Correlators", Phys. Rev. D 82 (2010) 034030 [Erratum-ibid. D 82 (2010) 119907]

[4] D. Greynat, P. Masjuan and S. Peris,"Analytic Reconstruction of heavy-quark two-point functions at $\mathrm{O}\left(\alpha_{s}^{3}\right)^{\prime}$, Phys. Rev. D 85 (2012) 054008.

[5] J. Siegrist et al., Hadron Production by $e^{+} e^{-}$Annihilation at Center-Of-Mass Energies Between 2.6-GeV and 7.8-GeV. Part 1. Total Cross-Section, Multiplicities and Inclusive Momentum Distributions, Phys. Rev. D26 (1982) 969.

[6] L. Criegee and G. Knies, Review of $e^{+} e^{-}$experiments with PLUTO from 3-GeV to 31-GeV, Phys. Rept. 83 (1982) 151.

[7] C. Edwards et al., Hadron production in $e^{+} e^{-}$annihilation from $s^{1 / 2}=5-\mathrm{GeV}$ to 7.4-GeV, . SLAC-PUB-5160.

[8] A. E. Blinov et al., The Measurement of $R$ in $e^{+} e^{-}$annihilation at center-of- mass energies between 7.2-GeV and 10.34-GeV, Z. Phys. C70 (1996) 31-38.

[9] CLEO Collaboration, R. Ammar et al., Measurement of the total cross section for $e^{+} e^{-} \rightarrow$ hadrons at $s^{1 / 2}=10.52-G e V$, Phys. Rev. D57 (1998) 1350-1358, [hep-ex/9707018].

[10] CLEO Collaboration, D. Besson et al., Observation of New Structure in the $e^{+} e^{-}$Annihilation Cross-Section Above B B̄ Threshold, Phys. Rev. Lett. 54 (1985) 381.

[11] D. Besson et al. [CLEO Collaboration] "Measurement of the Total Hadronic Cross Section in e+eAnnihilations below 10.56-GeV", Phys. Rev. D 76, 072008 (2007)

[12] BES Collaboration, J. Z. Bai et al., Measurements of the Cross Section for $e^{+} e^{-} \rightarrow$ hadrons at Center-of-Mass Energies from 2 to 5 GeV, Phys. Rev. Lett. 88 (2002) 101802, [hep-ex/0102003].

[13] M. Ablikim et al., Measurements of the continuum $R_{u d s}$ and $R$ values in $e^{+} e^{-}$annihilation in the energy region between 3.650-GeV and 3.872-GeV, Phys. Rev. Lett. 97 (2006) 262001, [hep-ex/0612054].

[14] CLEO Collaboration, D. Besson et al., Measurement of the Total Hadronic Cross Section in $e^{+} e^{-}$ Annihilations below 10.56 GeV, Phys. Rev. D76 (2007) 072008, [0 706 . 2813].

[15] CLEO Collaboration, D. Cronin-Hennessy et al., Measurement of Charm Production Cross Sections in $e^{+} e^{-}$Annihilation at Energies between 3.97 and 4.26 GeV, Phys. Rev. D80 (2009) 072001, [0801.3418].

[16] K. Melnikov and T. v. Ritbergen, "The Three loop relation between the MS-bar and the pole quark masses," Phys. Lett. B 482 (2000) 99.

[17] D. Greynat, P. Masjuan and V. Mateu, Work in progress. 